\title{
Epidiphtherische Bulbärlähmung.
}

\author{
Von \\ E. Trömner (Hamburg) und A. Jakob (Hamburg-Friedrichsberg). \\ Mit 4 Textfiguren und 1 Tafel.
}

Die pathogenetische Bedeutung der Infektionskrankheiten für die Neurologie beruht in ihrer Eigenschaft als pathologisch einheitliche Ursache und in der darauf gegründeten Möglichkeit, einen klinisch durchsichtigeren Kausalnexus zu erkennen, als es sonst in der Ätiologie organischer und funktioneller Neurosen möglich ist. Daß so gewaltige biochemische Revolutionen des gesamten Organismus, wie sie die Infektionskrankheiten darstellen, auch das Nervensystem in mannigfachster Weise in Mit- und Nachleidenschaft ziehen, ist ja ebenso bekann als begreiflich. Ich brauche nur an die prodromalen Cerebralerscheinungen vieler Infektionen, den initialen Herpes, brauche nur an den langen und bunten Heereszug der postinfektiösen Nervenleiden zu erinnern; ja, sogar ohne daß funktionelle Läsionen nachweisbar sind, pflegt sehr oft doch der Liquor spinalis Zeichen infektiöser Beeinflussung aufzuweisen, wie u. a. die Untersuchungen Schott müllers gelehrt haben. Während nun eine große Reihe von Infektionen äußerst mannigfache nervöse Nachleiden hinterlassen, wie z. B. Typhus, Malaria, Pneumonie, Pertussis, gründen sich andere postinfektiöse Neurosen auf eine mehr spezifische Affinität des Nervensystems zu jenem Virus. Wenn z. B. die Influenza mit Vorliebe sensible Nerven affiziert, die bekannten Neuralgien erzeugend, so befällt das Virus diphthericum fast ausschließlich motorische Nervenbahnen; ja, seine Vorliebe dafür ist so vulgär, daß3 die epidiphtherischen Lähmungen die zuerst bekannt gewordenen und beststudierten postinfektiösen Lähmungen überhaupt sind. Ihre ersten Beschreibungen stammen sogar bereits aus der Mitte des 18. Jahrhunderts, z. B. von Ghisi und Chomel (1749), Bard (1771) u. a. Schon diese ersten Autoren betonen die Häufigkeit gewisser typischer Lähmungsformen, welche auch durch zeitliches Auftreten und Prognose ein als einheitlich imponierendes Krankheitsbild darboten. Seitdem wurden Krankheitsbilder dieser Art mit zunehmender Häufigkeit gesehen und beschrieben. Ihre Häufigkeit beträgt nach Angabe deutscher Autoren etwa 5\% aller Diphtheritisfälle nach $\mathrm{Heubner,} 7 \%$ nach Baginsky, 8\% nach Nordmann, während auffallenderweise englische 
Autoren mehr als die doppelte Häufigkeit erlebt haben wollen; z. B. Goodall $11 \%$, Woodhead $14 \%$ und Rolleston sogar $22 \%$; die erstaunliche Differenz dieser Statistiken beruht wohl weniger auf einer größeren diphtherotoxischen Anfälligkeit dess englischen Nervensystems, als auf einer allzu weitherzigen Begrenzung des Begriffes Gaumenjarese.

Die übergrobe Mehrzahl jener Fälle bietet nun klinisch das Bild ciner vom Locus morbi an sich ausbreitenden Polyneuritis entweder in aufsteigender Form als Gaumen- und Schluckparese durch Glossopharingeus- und Vaguslähmung, innere oder äußere Augenm uskellähmung, oder in generalisierter Form als allgemeine distale Polyneuritis.

Sehr selten sind diesen bekannten Fällen gegenüber solche Lähmungskomplexe im Bereich der Hirnnerven, welche mehr als Bulbärläh mung denn als multiple Neuritis der Hirnnerven bezeichnet werden müssen, Fälle, welche auch durch einen von dem benignen (tros der gewöhnlichen Fïlle abweichenden infaust progredienten Verlauf eine Sonderstellung rinnehmen, welche also bulbür enden, verschieden von denjenigen, welche an Herzschwäche infolge von Degeneration des Myocard oder des Vagus zugrunde gehen. Rein bulbäre Lähmungskomplexe derart nach Diphtheritis sind so selten, daßs selbst Oppe nheim die wenigen bisher beschriebenen, z. B. von Harris und T'inel-siredey als noch ätiologiseh unaufgeklärt bezeichnet. Diese Seltenheit mag es rechtfertigen, als Gabe zu dieser Festnummer einen klinisch und anatomisch determinierbaren Fall dieser Art beizustenem, welcher längere Zeit in der Hamburger Allgemeinen Poliklinik beobachtet und dessen finale Krankengeschichte später von Herm Dr. Nonne, auf dessen Abteilung die Patientin zum Exitus kam, uns ebenso wie das autoptiseh gewonnene Material freundlichst zur Bearbeitung ïberlassen wurde.

W'ir lassen zunächst die Krankengeschichte folgen:

L. G., 8 Jahre alt, war insofern Trägerin einer gewissen Disposition, als eine rehwester von ihr dureh Diphtheritis herzleidend, ein Bruder 9 Jahre früher an Diphtheritis gostorben war und der Vater an Asthma leidet. Sie war ein zartes, jahrelang skrofulöses Kind, welches im 2. Lebensjahre an Stimmritzenkrampf litt und im 3. Jahre ein ,verstecktes Scharlitch" durchmachte. In der katholischen Gemeindeschule angesteckt, bekam sie im Juli 1911 Diphtheritis mit hohem Fieber, welches ctwa 9 Tage lang andauerte. Während dieser Zeit lag sie immer für sich weg, phantasierte nachts und mußte mit Gewalt gefüttert werden. 14 'Tage lang bestand graner Belag im Hals, am Körper war sie empfindlich, schrie, wem sie angefa $\beta$ t wurde. Am 5. Tage erhielt sie von J)r. M. cine Serumeinspritzung, welche zunächst bessernd zu wirken schien; als sie am 20. Tage nach der Einspritzung aufstelen wollte, konnte sie kium gehen, hatte jedoch keine Zeichen von Lähnungen. Anfang Sieptember, also 6 Woehen nach der Lrkrankung, bremerkte die Mutter eine Mehlaffheit des (iesichts, so daß sie zu weinen schien, weun sie lachen wollte. Einige Wochen später begann sie sich öfter durch die Nase zu verschlucken. Mitte Oktober wurde sie unserer Allgemeinen Poliklinik zugeführt, wo zunächst nur cine leichte Schwäche der Lidheber, der lacialismuskulatur (ohne BAR.) and der (taumenheber fost gestellt wurds. Dir iibrigen 
Funktionen, vor allem Rumpf und Glieder, waren normal. Wir galvanisierten das Kind regelmäßig, ließen es viel ruhen, ab und zu schwitzen und reichlich ernähren. Ganz allmählich aber verschlechterte sich das Bild, so daß Anfang Dezember folgender Status notiert wurde:

Doppelseitige Halbptosis, Pupillen norma!, Lichtreaktion etwas gering, ('ornealreflex schwach; Augenbewegung: Linkes Auge richt ganz nach innen gewendet, Aufwärtswendung beiderseits unvollkommen; (Aaumen- und Rachenreflex schwach, Masseterenreflex positiv.

Augenendstellungen unsicher erreicht und nicht lange festgehalten.

Stirnhebung 0, Brauenrunzeln Spur, Lagophth. mittleren Grades beiderseits, Oberlippenhebung gering, Zunge positiv, Gaumenhebung schwach, Kopfbewegungen wenig kräftig, Glieder leidlich kräftig, Reflexe, Sensibilität und Koordination crhalten.

Elektrisch bestand im Facialisgebiet: faradische lirregbarkeit positiv; galvanisch quantitative Herabsetzung ohne EAR.; nur im liontalis ctwas verlangsamte Zuckung.

Aussprache dysarthritisch verwaschen, jedoch verständlich.

Am 12. Dez. trat zuerst ein leichter Erstickungsanfall infolge Versehluckens und ungenügender Expektoration auf. Zugleich waren die Augen zu 3/4 ptotisch geschlossen und die Gesichtsinnervation eine äußerst schlaffe. Das Kind wurde jetzt nach Eppendorf verlegt auf die Abteilung von Herm Dr. Nonne, welcher uns die weitere Krankengeschichte, das Sektionsprotokoll und das (iehimpräparat freundlichst zur Verfügung stellte.

In Eppendorf wurde am 12. Dez. notiert: $A b$ und zu blitzartige Zuckungen der Extremitäten und der Gesichtsmuskulatur, beiderseits Ptosis des oberen und unteren Augenlides, Parese des rechten Occulomotorius, besonders des internus, Stimfacialis beiderseits spurweise innerviert, Zunge und Kaumuskeh schwach innerviert, Kopfbewegungen sowie Rumpfaufrichten schwach. Der übrige Befund deckte sich mit dem von uns erhobenen. Die Frstickungsanfälle, infolge von Verschlucken des eigenen Speichels, wiederholten sich immer häufiger, und unter zunehmender Herzschwäche erfolgte am 31. Dez. der Tod.

Die am nächsten Tage vorgenommene Autopsie ergab als direkte Todesursache Bronchopneumonie des rechten Unterlappens. Daneben fand sich Schwellung der Mesenterialdrüsen und Cyanose sämtlicher inneren Organe. Anatomisch waren die Organe normal und das Blut, bakteriologisch geprüft, steril.

Diese Krankengeschichte enthält eine Reihe von interessanten Momenten. Zunächst scheint die Tatsache, daß zwei Geschwister in verschiedenen Jahren vorher an Diphtheritis starben resp. litten, auf eine gewisse familiäre Disposition schließen zu lassen; ein Moment, welches Hand in Hand mit einer anfälligen Konstitution offenbar die Prognose der Erkrankung bestimmte.

Die am 5. Tage erhaltene Serumeinspritzung hat zweifellos weder geschadet noch genützt, denn alle kritischen Verehrer des Behringschen Serums machen einstimmig seine Einverleibung am 1. oder spätestens 2. Tage zur Bedingung einer einwandfreien Wirkung. Einspritzungen am 4. und 5. Tage sind sicher in jeder Form wirkungslos. Allerdings hat Baginsky mitgeteilt, daß vor der Serumbehandlung auf 993 Fälle seiner Erfahrung 68 Lähmungen, also 7\%, nach seiner Einführung aber auf 525 Fälle 27 Lähmungen, also $5 \%$ gekommen seien; aber bei der 
Deutung solcher Statistiken sind, wie häufige analytische Diskussionen der Di.-Statistiken in den letzten Jahren gemahnt haben, doch so viele Momente zu berücksichtigen (verschiedene Schwere der Epidemien, Bedingungsänderungen der Finlieferung ins Krankenhaus, Ungleichwertigkeit des Materials $\mathbf{u}$. a.), daß Abweichungen der Resultate späterer nicht wunder nehmen. Slawyk z. B. sieht nur eine unerhebliche Abnahme der Lähmungen nach Einführung des Serums, etwa 0,2\%, Wollacot meint sogar, eine gewisse Zunahme gesehen zu haben, eine Zunahme, welche er auf bessere Konservierung schwerer Fälle durch das Serum schiebt. Unser Fall spricht jedenfalls weder gegen noeh etwa für die Meinung, daß das Serum eine Lähmung verhindernde Wirkung ausübe (Comby).

Das klinisch Bemerkenswerte unseres Falles ist nun die Beschränkung a uf die motorischen Hirnnerven. Die allgemeine Schwäche beim Versuch, nach dem 20. 'Tage das Bett zu verlassen, ist keinesfalls als Zeichen peripherer Extremitätenlähmung zu betrachten, da sie sich bald wieder verlor, und da vor allem bei der ersten Untersuchung Mitte Oktober sämtliche Extremitätenreflexe normal waren; Verlust der Sehnenreflexe aber gewöhnlich die erst erscheinende und zuletzt verschwindende Manifestation neuritischer Extremitätenlälımung zu sein pflegt.

Die wahre Fröffnung des gesamten Lähmungskomplexes bilden vielmehr Paresen im gesamten Gebiet des Facialis, welchen sich bald auch geringe Ptosis durch Oculomotorius-Parese anschloß. Ob diese Lähmung peripheren oder pontinen Ursprungs, würde, da doppelseitige periphere Facialislähmung bei langsamer Entwickelung und progressiver Tendenz bekanntlich äußerst selten sind, mit leichterem Herzen im Sinne der pontinen Ätiologie zu beantworten sein, wenn nicht diese seltenen Fälle eben relativ häufig bei der Di. vorkämen, bei deren Lähmungen Beginn mit Faeialislähmung wohl cine Raritä, aber kein Unikum darstellt. Eine Statistik von Baginsky nennt z. B. unter 68 Lähmungen überhaupt zweimal Facialislähmung, eine andere später mitgeteilte unter 61 sechsmal Gesichtslähmung. Auch Mangel ausgesprochener Entartungsreaktion spricht weder für noch gegen den einen oder andern Ursprung, wenn auch periphere Neuritis viel regelmäßiger zur Entartungsreaktion führt.

Nur ein gewisses Maß von Wahrscheinlichkeit darf daher unsere Annahme beanspruchen, wenn wir aus dem subakuten Beginn, der langsamen Progression, der Doppelseitigkeit und dem Ergriffensein sämtlicher Zweige, bei Fehlen vollkommener Entartungsreaktion und bei Mitbeteiligung anderer motorischer Hirnnerven folgern, daß es im wesentlichen zentrale Prozesse sind, wenn auch nicht ausschließlich, welche schon in der initialen Prosopoparese zum Ausdruck gelangen. Das 
weitere Fortschreiten des Prozesses auf andete Hirnnerven scheint freilich die pontine Ursprungshypothese wieder nicht zu stützen, da der dem Facialis nächst benachbarte Kern des Abducens fast völlig freiblieb - nur geringe Schwäche der Seitwärtsbewegung bestand - , und da auch die Zunge, welche bekanntlich bei den chronisch progressiven Formen der Bulbärparalyse, auch des Kindesalters, von welchen Trömner ein selten gebliebenes Beispiel beschrieb, ein hauptleidtragender Teil ist, hier erst in den letzten Wochen geringe Schwäche zeigte. Der Facialislähmung schloß sich sehr bald eine (Y्रlossopharyngeus(Schluck-)Lähmung an, dann folgten Oculomotoriusparesen und schliel3lich leichte Schwäche im Abducens, motorischen Quintus, der Zunge und den Hals- und Kopfmuskeln, während sensible Nerven völlig freiblieben; also ein Lähmungssyndrom, dessen Fortschreiten sich nicht nach anatomischen Wegen, weder pontiner noch peripherer Erkrankung, richtet, sondern welches als Folge einer elektiven Wirkung der pathogenen Noxe begriffen werden muß. Es liegt also weder eine Neuritis ascendens im Sinne Webers, Senators, Leydens vor, noch eine in bestimmter Richtung fortschreitende Kernerkrankung, sondern ein durch bestimmte Neuroaffinität des Diphthero-Toxins bedingter Lähmungskomplex. Fine solche elektive Wirkung reigt das Toxin ja auch bei Lähmung der Extremitätennerven, welche zuerst und am längsten die reflexvermittelnden Fasern der Nerven resp. die Reflexcentren im Rückenmark ergreift. Patellarreflexe können noch fehlen, wenn längst die Motilität der Nerven wieder hergestellt ist. In der 'Tat sind periphere Nerven sonst in der Mehrzahl der Fälle mit affiziert. Fehlen der Patellarreflexe nimmt z. B. Remak in etwa 50\% aller Fälle von diphtherischer Gaumenlähmung an, Bernhard fand sie unter 21 Fällen sogar 20 mal fehlend, und auch $Z$ appert bezeichnet sic als nahezu regelmäßig erloschen, Meinungen, die nebenbei - nach unsren Erfahrungen - zu weit gehen. In unserem Falle dagegen blieben die Patellarreflexe erhalten und haben wahrscheinlich niemals gefehlt. Frühe Ptosis wie in unserem Falle wird sowohl von Re mak als auch von Wilbrandt-Saenger, welche als ihre Ursache Kernaffektion annehmen, als nur vereinzelt vorkommend erwähnt.

Den unseren ähnliche, nur auf motorische Bulbärnerven beschränkte Lähmungsbilder sind bis jetzt sehr selten beschrieben. Oppenheim erwähnt einen ähnlichen Fall von Tinel - Sirede y, dessen Original mir nicht zur Verfügung stand, und als zweiten einen von Harris beschriebenen, wo bei einer 24 jährigen Frau einige Wochen nach der Infektion Schluck-, Stimm- und Facialislähmung bei ebenfalls erhaltener galvanischer Erregbarkeit und Bestehenbleiben der tiefen Reflexe eintrat. Oppenheims Zweifel an seiner diphtherischen Natur kann ich mich im Hinblick auf unseren Fall nicht mehr anschließen. 
Ein für die Annahme bulbärer Grundlage sprechender İmstand scheint mir auch die große Ähnlichkeit unseres Falles mit der myasthenischen Bulbärlälımung zu sein. Die subakute Entwickelung, die Kombination von Facialis- mit Oculomotoriuslähmungen, mit Dysphagie und Dysarthrie, die Schwäche der Kopf- und Rumpfinnervation, der Mangel von Entartungsreaktion, Atrophien und Reflexanomalien: allo diese Zeichen, welche speziell Oppenheim, einer ihrer besten Kenner, als charakteristisch für Myasthenie anführt, zeigt unser Fall.

Auch unsere Patientin bot übrigens gesteigerte Ermüdbarkeit in Augen-, Gesichts- und Zungenbewegungen, wenn auch lïngst nicht in der Intensität, wie sie als motorische und elektrische myasthenische Reaktion bekannt ist. Immerhin ist die äußere Ähnlichkeit so groß, daß unser Fall, als zweifellos toxischer, geeignet ist, auch der autotoxischen Theorie der Myasthenie eine wesentliche Stütze zu leihen. Früher, als die anatomische (xrundlage der diphtherischen Lähmungen noch fehlte, wurden übrigens sogar sie zu den asthenischen Lähmungen im Sinne Gublers gerechnet, bis 1862 Charcot und Vulpian ihre „dégénérescence granulo-graisseuse " in den Gaumennerven fanden.

Zu allem U̇berfluß erwähnen wir noch, daß bei Myasthenie z. T' auch ähnliche pathologisch-anatomische Befunde erhoben wurden.

Die aus dem klinischen Bilde gewonnenen Vermutungen, daß das lähmungssyndrom die Folge einer elektiv toxischen Wirkung ist, welches die Bulbärkerngegenden zwar hauptsächlich. zum kleineren Treil aber auch die peripheren Nerven ergriffen hat, dise Mutmaßungen erhalten nun ihre Stijte durch das Resultat der anatomischen Untersuchung. Infolge eines Versehens bei der Autopsie wurde uns leider nicht das gesamte Material zur Verfügung gestellt, sondern nur Stücke des Nervus vagus, Facialis, Radialis und die Medula oblongata vom Austritt des Glossopharyngeus mit dem ganzen Rückenmark. (Alkohol- und Formolfixierung.)

Dic im Laboratorium der Staatsirrenanstalt Friedrichsberg vorgenommene mikroskopische Untersuchung (Takob) ergab folgendes:

Der Quersehnitt der Medulla oblongata zeigt zunächst in Zell- und Faserpräparat keine gröberen Ausfälle; mit der Weigertschen Markscheidenfärbung sind keine Degenerationen in den intramedullär verlaufenden und austretenden Nerven nachzuweisen; insbesondere erscheinen zunächst die Nin. Vagi, Gilos:opharyngei und Hypoglossi intakt.

Das Toluidinblau-Bild jedoch läßt in diesen (iebieten deutliche pathologische Veränderungen crkennen. An zahlrcichen kleinen und größeren Gefäßen des Querschnitts fällt eine meist nur geringgradige. Infiltration mit Lymphocyten auf, die einen deutlichen plasmatischen Hof besitzen (Polyblasten Maximows). An manchen Stellen sind dio infiltrutiven Frseheinungen ibusgesprochener, wie aus dem Mikrophotogramm der 'Textfigur 1 erhellt. In Lumen dieses (Gefäßes erkennt man die Ansammlung zahlreicher polynucleärer Leukocyten, ein Befund, der an vielen Blutgefäßen noch deutlicher wird, so daß oft der ganze Querschnitt von polynucleären Leukocyten vollgepfropft ist. Die Endothelien, vornehmlich die 
der Capillaren, sind geschwellt, seltener trifft man Sprossungen und Kernteilungsfiguren in den Gefäßwandzellen.

All cliese Frscheinungen sind am Boden der Ra u te ngrube am deutlichsten. Un die Gefäße mittleren und größeren Kalibers sind die Lymphräume in außergewöhnlicher Weise erweitert und mit geronnenor Lymphe erfïllt, die nicht selten entzündliche Elemente, wie polynueleäre Leukocyten oder plasmatische Lymphocyten enthält; solche Zellen liegen jedoch nur vereinzelt in dem ausgedehnten

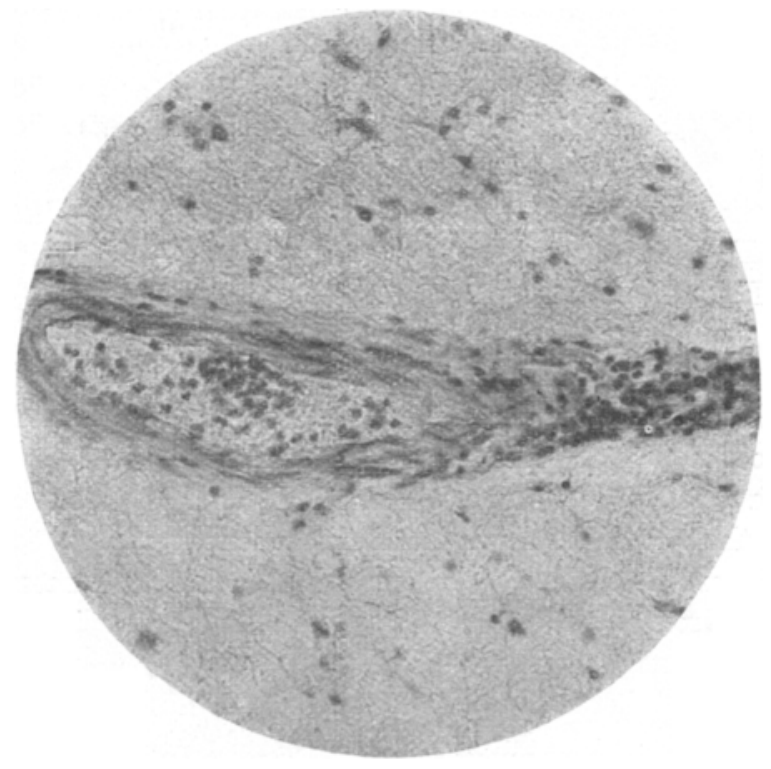

Fig. 1. Gefäb ans der Med. oblong. Infiltrution mit Polyblasten. In Lumen zahlreiche polynucl. Leukocyten, Toluidinblaupräparat. - Mikrophotographie.

perivasculären Raum. Diese Verhältnisse stellen sich sehr schön in v. Giesonoder modifizierten Mallory-P'räparaten dar (vgl. T'extfigur 2, die ein Gefäß aus dem motor. Glosso pharyngeus-Kern mikrophotographisch wiedergibt).

Es ist auffallend, wie gerade die nervösen Elemente, welche in der Nachbarschaft solcher Gefäße liegen, pathologisch verändert sind. Hier trifft man sehr häufig Ganglienzellen von abgerundeten lormen und randständigen Kernen, deren Chromatinschollen sich undeutlich färben. Was in allgemeinen die Ganglienzellen der im verlängerten Marke gelagerten motorischen Korne angeht, so zeigt das Zellpräparat bei schwacher Vergrößerung keine schwereren störungen. Erst mit stärkeren Linsen erkennt man in jenen Kernen zahlreiche deutlich degenerierte Zellformen. Sie färben sich dunkel mit verklumpten Chromatinschollen, sind fortsatzarm und zeigen den ebenfalls dunkel tingierten Kern exzentrisch verlagert; auch wabig entartete (ianglienzellen befinden sich darunter. 1)ie (Alia der Umgebung ist in leichter Wucherung begriffen und 'nthält (bei der Herxheimerschen Scharlachfärbung) feine rote Tröpfchen in ihrem Plasma. Derartige Veränderungen sind vornehmlich in dem Nucleus hypoglossus, glossopharyngeus-vagus und dem Rollerschen Kern der beiden Seiten nachzuweisen.

Neben den feineren Strukturveränderungen lisssen sich schließlich, wenn auch seltener, noch gröbere Störungen erkennen, welche in Form älterer und 


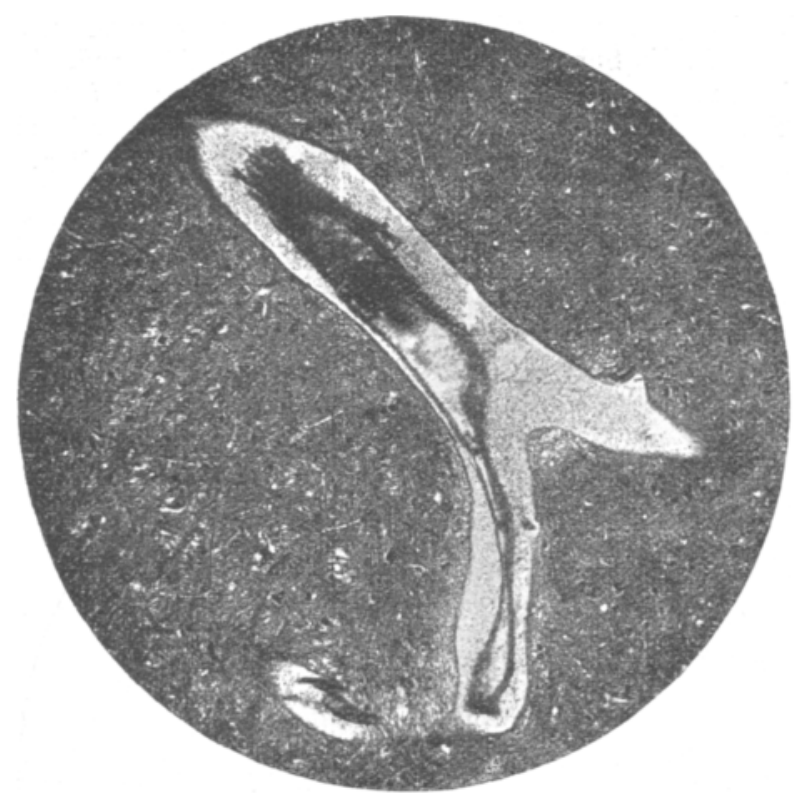

Fig. 2. Getial. aus dem motor. Gilosso-pharyng. Kern mit erweiterten und nit patholog. lymphe angefüllten perivase. Raum. Modif. Mallory-Firlung. - Mikrophotographie.

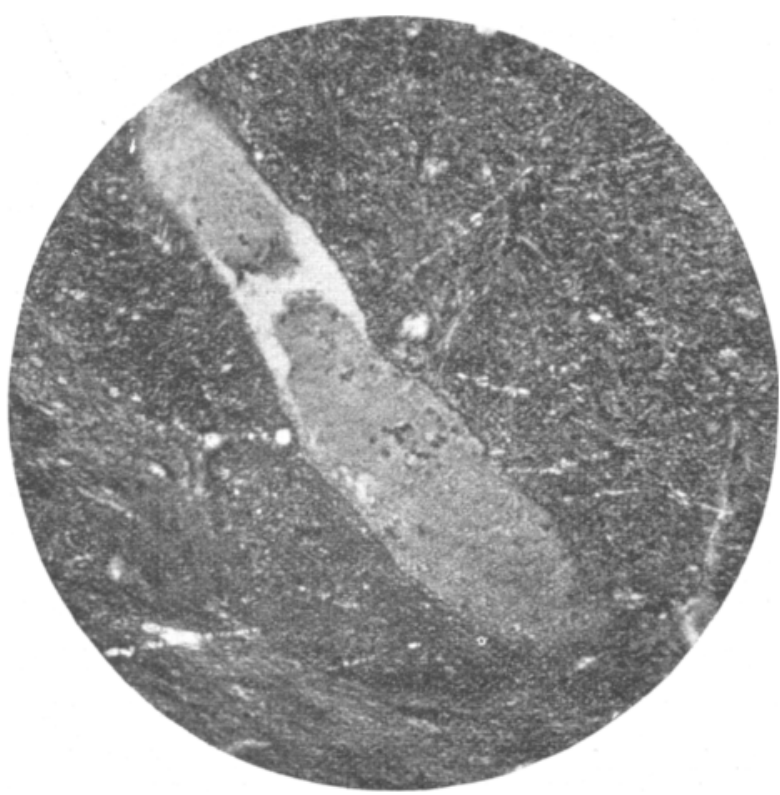

Fig. 3. Frischer Blutherd ventral von Xincl. 1X, X. Mudif. Hallory-Härbung. - Mikrophotographie. 
frischer Blutungen gleichfalls in jenen Gegenden lokalisiert sind. So treffen wir (vgl. Textfig. 3) etwas ventral vom Glossopharyngeus-vagus-Kern, in manchen Schnitten noch in die Substanz des Nervenkernes hineinragend, einen größeren hämorrhagischen Herd mit zum Teil bereits ausgelauchten und deformierten roten 13lutkörperchen, polynucleären Loukocyten und vereinzelten, am Rande gelegenen Makrophagen, welche in ihren Vakuolen Blutelemente enthalten. Eigentliche Körnchenzellen sind hier nicht zu finden.

In der Nachbarschaft dieses zweifellos friseheren Blutherdes trifft man kleino gliöse Narben mit hin und wieder eingestreuten Körnchenzcllen, Befunde, die wohl als Residuen capillarer Apoplexien \%u deuten sind. Ferner begegnet man noch in diesen Partien kleinen Höhlenbildungen (vgl. Textfigur 4), die von einem Glia-

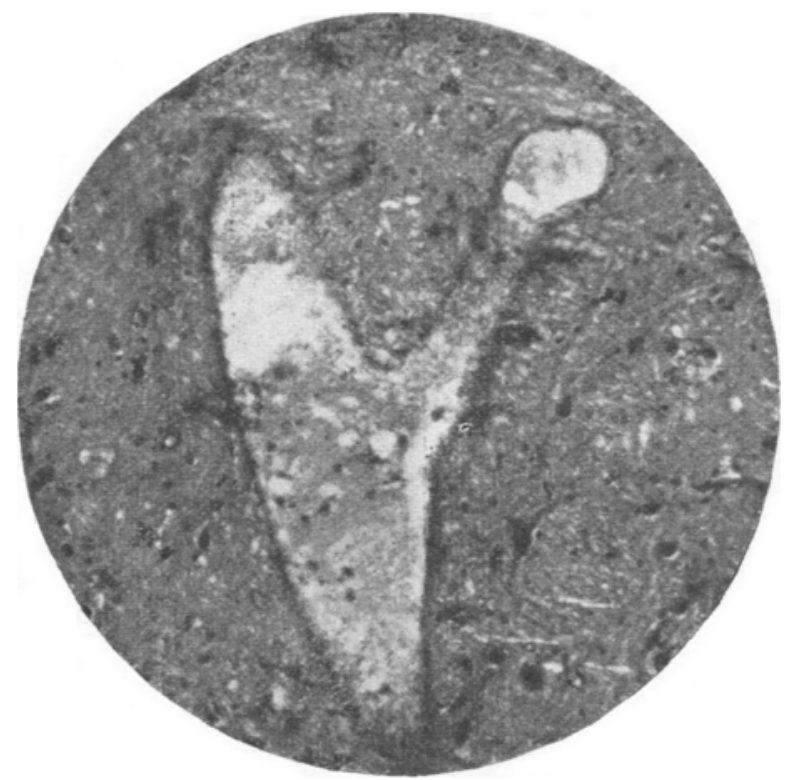

Fig. 1. Hämorrhagische Höblenbildung, von starkem Gliafilz ungeben. I. o. zahlıciche Riesengliazellen. Boden der Rautengrube. Modif. Mallory-Färbung. - Mikrophotographie.

filz umgeben, stellenweise von feinen, sich vielfältig durchkreuzenden Gliafasern überwuchert werden; in ihrer Umgebung liegen stark gewucherte, faserbildende Gliazellen, wie sie auch in der beigegebenen Textfigur 4 namentlich links oben, gut zu erkennen sind. Stellenweise sind diese Höhlen-bildungen - wie auch in der Textfigur 4 - von Gerinnsel, Resten von Blutelementen, selten auch von Körnchenzellen angefüllt.

Es muß betont werden, daß sich all diese Schädigungen in der Gegend der erwähnten Nervenkeme lokalisiert finden, und daß trotzdem die austretenden Gehirnnerven, insbesondere die IX., X. und XII., keine nachweisbaren Degenerationen zeigen. Auch im Cervicalmark fehlen sekundäre Degenerationen oder gröbere Veränderungen der grauen Substanz, und nur an seinen Gefäßen sind entzündliche Erscheinungen in den erweiterten Lymphräumen deutlich ausgesprochen. Die Meningen zeigen sich überall frei von infiltrativen Elementen.

Auch die peripheren Nerven, die zur Untersuchung gelangten, zeigen 
ähnliche Veränderungen, welche ihrer pathologisch-histologischen Natur nach ïberall im wescntlichen gleich, nut sich in der jeweiligen Ausdehnung des Prozesses verschicden gestalten. An hochgradigsten ist das Perincurium affiziert. Das gauze (iewebe erscheint intensiver gefärbt, von zahlreichen Rundzellen durchsetzt, Veränderungen, welehe besonders deutlich in der Umgebung der Gefäße hervortreten. Dic (iefäßwandzellen (Taf. 1, Fig. 1) sind geschwollen, einige Elemente der Adventitia zu Körnchenzellen umgewandelt. Die ganze Nachbarschaft solcher (Gefäbe ist angefüllt mit zum T'eil stark gewucherten Fibroblasten und einer Menge kleiner Zellen, die sich in ihrer Erscheinungsform zum Teil als lymphocytäre Blutelemente, zum Teil als Polyblasten charakterisieren. Häufig begegnet man Makrophagen mit Blutpigment und ausgesprochenen Fettkörnchenxellen. Hinzelue Stellen des Perineuriums sind mit frischeren und älteren Blutaustritt ro nebst den entsprechenden reaktiven Abbawelementen durehsetzt.

Liter den innersten Lamellen des Perineuriums sammeln sich mancherorts srößere Mengen einer pathologisehen Flässigkeit an, welche bei Formolfixierung seronnen, sich mit den Malloryschen Farben als eine blaßblauc, zarte, feinkörnige oder wabjige Masse darstellt. In ihr liegen ganz zerstreut polynucleäre Leukocyten, Polyblasten, und, seltener, fettgefüllte Körnchenzellen. Ebenso häufig int das Endoneurium von dieser pathologischen Lymphe durchtränkt und in seinen Maschen erweitert, und nicht selten ist eine mitunter recht erhebliche $\mathrm{I}_{4} \mathrm{y}$ m phor. rhagir in das Nervemparenchym selbst eingetreten. Fig. 2 auf Taf. I zeigt einen solchen Befund: dic nervösen fasem sind auf cine weite Strecke völlig zerstört durch das Eindringen dieser Flissigkeit, in der wueh hier ganz zerstreut die genannten Zellformen zu sehen sind. Namentlich kleine Lymphocyten und mehrkernige Leukocyten sind noch in weiterer Umgebung zwischen den aufgelockerten Norvenfasern amzutreffen, welch letztere dann in verschicdenen Stadien der J)egeneration begriffen sind.

Ftellenweise zeigen auch die Gefäße des Endoneuriums ähnlich entzüulliche Kr. seheinungen. In Fig. :3, 'Taf. I sehen wir ein solches Gefäß vollgepfropft mit polynucleären Leukocyten und plasmatischen Lymphocyten (vielleicht als eine Art Thrombenbildung aufzufassen); das angrenzende Nervengewebe ist ron der pathologischen Tymphe aufgelockert, in welcher sich gerade an einer Stelle zahlreiche polynucleäre Leukoeyten angesammelt haben.

B.i alledem bleiben die Hauptveränderungen in peripheren Nervensystem auf dits Perineurium beschränkt. Das Nervenparenchym ist nur in einzelnen Nervenbündeln schwer geschädigt, so daß es hior - an einem Nervenbündel im X. facialis -- zur Ausbildung cines größBeren Gebietes von Kornstrangfasern gekonmen ist. Sonst sieht man nur hin und wieder eingestreute Kernstrangfasern und Nervenfasern in verschiedenen Stadien der Wallerschen Degeneration.

Das Epineurium zeigt außer siner oft außerordentlichen Anhäufung von Fettzellen nichts Besonderes.

Was die Fxtensität des Prozesses angeht, so sind die Veränderungen am Facialis und Vagus am meisten ausgesproehen, während der $N$. radialis nur weniger, jedoch im gleichen Simne, befallen erscheint. Auch dic bei der Untersuchung des Rückemmarks getroffenen peripheren Nervenabschnitte lassen stellenweise ähnliche Affektionen erkennen, namentlich die im Hals- und Brustmark austretenden Nervenstämme. So ist Fig. 3 auf 'Taf. I einem im oberen Brustmark entspringenden Nervenbündel entnommen. Sehließlich bedarf es noch der Frwähnung, daß die vorderen und hinteren Wurzeln des ganzen Rückenmarks normal erscheinen.

Resumieren wir kurz die gewonnenen, in obigem kurz skizzierten Resultate, so bleibt es zunächst bedauerlich, daß die Untersuchung infolge der geringen Menge des Materials, das uns zur Verfügung gestellt 
w urde, eine lückenhafte bleiben mußte. So konnten die Kerne des Facialis wie auch die der Augenmuskel histologisch nicht durchforscht werden, wie ja auch die kleinen, nur in Formol fixierten Stückchen der peripheren Nerven keineswegs eine völlig erschöpfende histologische Behandlung gestatteten. Immerhin sind die erhobenen Befunde so charakteristisch und in ihrer pathogenetischen Auffassung so eindeutig, daß3 sie uns ein ganz gutes anatomisches Bild dieses kliniseh so interessanten Falles geben.

Es handelt sich in der Hauptsache um entzündliche Veränderungen, die sich auf die Gefäße und deren Umgebung beschränken und so offenbar im gleichen Sinne das zentrale wie periphere Nervens yste m, freilich nur in ganz bestimmter Lokalisation, befallen haben. Anatomisch ist es schwer zu entscheiden - namentlich mit Rücksicht auf die Lückenhaftigkeit der Untersuchung - welcher der verschieden lokalisierten Prozesse für den funktionellen Ausfall höher $\mathrm{zu}$ bewerten ist; im zentralen wie im peripheren Nervensystem sind die Veränderungen nicht allzu hochgradige, immerhin doch so schwer, daß sie auch jede für sich klinische Störungen heraufbeschwören mußten. Bei voller Würdigung der Schwierigkeit eines solchen Vergleiches überhaupt will es scheinen, daß die zentrale Affektion in diesem Falle doch ausgesprochener war als die periphere, namentlich was die Schädigung des nervösen Gewebes selbst angeht.

Das eine aber wird sich mit Sicherheit aus der Eigenart des anatomischen Bildes schließen lassen, daß der pathologische Proze $ß$ - rein toxischer Natur, wie durch Brieger und Fränkel experimentell nachgewiesen ist - beide Teile des Nervensystems im gleichen Sinne, jedoch unabhängig voneinander, befallen hat.

Bekanntlich ist die bereits häufig diskutierte Frage nach der pathogenetischen Grundlage der postdiphtherischen Lähmungen entsprechend der Verschiedenartigkeit der jeweiligen histologischen Befunde ganz widersprechend beantwortet worden. So wurden von zahlreichen Autoren (u. a. von Charcot und Vulpian, Lorain und Lepine, Bailly, Schech, P. Meyer, Geyer, Spieler, Wladimirow) entweder ausschließlich oder doch vorwiegend peripher-neuritische Veränderungen gefunden und eine periphere Natur der Erkrankung postuliert. Gestützt wurde diese Ansicht durch die in vielen Fällen klinisch gemachte Lrfahrung von dem Überwiegen der Lähmungserscheinungen gerade auf der Seite, auf welcher die diphtherische Primärerkrankung allein oder intensiver lokalisiert war (Greenhow, Acker, Leyden, u. a.); einen weiteren Beweis für diese Ansicht läßt sich in jenen Beobachtungen von Hautdiphtherie erblicken, in denen der Ausgangspunkt der ihnen folgenden generalisierten Lähmungen genau der Lokalisation der Hautdiphtherie entspricht, Fälle, ,,wie sie Paterson - zunächst Lähmung 
des rechten Armes, dann generalisierte Lähmung nach Hautdiphtherie am rechten Zeigefinger - , Pitres und Veillard -- sensible Störungen der linken oberen Extremität vor der generalisierten Lähmung nach Hautdiphtherie der linken Infraclaviculargegend -, Ku Bmaul Bauchmuskellähmung, dann erst Lähmung der unteren Extremitäten nach Tiphtherie der Nabelgegend" - (cit. nach spieler) beschrieben haben.

Den Experimentalbeweis für diese Auffassung führte Babonneix, welcher durch Injektion kleiner Diphtherie-Toxindosen in die Ischiadicusgegend an Hunden, Kaninchen und Meerschweinchen zunächst isolierte, auf den Inoculationsort beschränkte, dann auch von hier fortschreitende allgemeine Lähmungen erzeugen konnte; daß es sich dabei nicht etwa um eine traumatische Neuritis handelte, ließ sich dadurch zweifellos erweisen, daß die Iähmung bei immunisierten 'Tieren ausblieb. Histologisch fanden sich entzündliche Veründerungen an den peripheren Nerven ,fonte granuleuse des fibres totale ou presque totale“" weiterhin einseitige Degenerationen der hinteren Wurzeln auf der eingespritzten Seite, der Spinalganglien, des ,faisceau sensitiv-réflexe" im Hinterstrang, außerdem noch Schwellung und (hromatolyse, seltener auch Atrophie der Ganglienzellen des Rückenmarks. Babonneix glaubt diese Befunde in Sinne einer aszendierenden Neuritis auffassen zu dïrfen.

Fino gewisse experimentelle Beweiskraft dürften auch die Befunde Spielers beanspruehen, der in Frühstadien der Frkrankung bereits eine schwere interstitielle wie parenchymatöse Fntzündung der Nervi vagi bei jeglichem Mangel pathologischer Veränderungen im Centralnervensystem feststellte, und der daraus den Schluß zog, daß ,der plötzliche, postdiphtherische Herztod seine Erklärung durch den Nachweis frühzeitiger peripherer Vagusdegeneration findet, und daß die peripherneuritische Natur die gewöhnlichste und häufigste klinische Form der postdiphtherischen Lähmung ist".

Eine große Reihe anderer Autoren hat aber — wiederum gestützt auf die Eigenart ihrer Befunde - cine vornelımlich centrale Natur der meisten diphtherischen Lähmungen angenommen, indem die einen von ihnen in einer Alteration des Gefäßsystems und seines Inhalts das Primäre erblickten, die anderen aber die toxisch-entzündlichen Veränderungen der centralen Nervensubstanz selbst für dic direkt ausgelöste und im Vordergrund stehende Schädigung hielten. So fand Buhl neben capillaren Blutungen im Gehirn, die Spinalwurzeln u. -ganglien verdickt, durch Blutaustritt verfärbt, stellenweise gelblich erweicht und von Exsudatmassen umschlossen, ,diphtheritisch infiltriert“. Ähnliche Verinderungen beschrieb R. Maier bei einem 6 jährigen Knaben, der bulbïre und Extremitätensymptome gezeigt hatte und rasch starb: in 
der Medulla oblongata, den Hirnstielen, den basalen Hirnganglien, dem oberen Halsganglion, dem Ganglion plexiforme und solare zeigten sich neben bald stärkerer, bald geringerer Anhäufung von lymphatischen Elementen in der Pia überall Gefäßectasien, Thrombenbildung und größere und kleinere hämorrhagische Herde.

Örtel fand in einem Falle von diphtherischer Lühmung neben Atrophie und Fettdegeneration der Muskeln im Gehirn, Rückenmark und peripheren Nerven zahlreiche capillare und größere Blutungen und zellreiche Exsudate und Infiltrate in der grauen Substanz des Rückenmarks; das Lumen des Centralkanals war durch ein zellreiches Exsudat ganz verstopft. Ähnliche Veränderungen sahen auch Da ma schino und Roger und Landouzy und erblickten in den Gefäßverïnderungen die Ursache der gleichzeitig beobachteten Fintartungen der vorderen Rückenmarkswurzeln und der peripheren Nerven.

Auch Pierret beschrieb in der Medulla oblongata und spinalis zerstreute fibrinöse und eiterige Infiltrationen der Pia; die Gefäßsscheiden und Nervenwurzeln waren mit zahlreichen Leukocyten infiltriert; stellenweise war es bis zur Thrombosierung kleiner Venen gekommen. Gerade mit Rücksicht auf diesen Befund erklärte Sanclair den von ihm als Meningolymphite bezeichneten entzündlichen Prozeß als die pathologisch-anatomische Grundlage der postdiphtherischen Lähmungen, der infolge von Diphtheritis in den Nervenseheiden, in den Gefïßscheiden und überhaupt in den lymphatischen Räumen des Nervensystems Platz greifen könne.

Schon vorher hatte Déjérine in 5 Fällen postdiphtherischer Lähmung neben einer parenchymatösen Degeneration in den vorderen Rückenmarkswurzeln in den spinalen Vorderhömern eine interstitielle und parenchymatöse Entzündung beschrieben, die er als Tephro(Polio-)myelitis anterior auffaßt, und in welcher er das Primäre bei der diphtherischen Lähmung sucht. Déjérine und Barth publizierten später noch einen dem Pierretschen in gewissem Sinne ähnlichen Befund, bei dem die Sektion eine eitrige Meningitis ergab, welche Bulbus und Halsmark von der Hypoglossuswurzel bis zum zweiten Cervicalnerv umgab. Das $1 / 2 \mathrm{~mm}$ dicke eitrig-fibrinöse Exsudat umfaßte die Wurzeln der Vagi, Glossopharyngei, Accessorii und Hypoglossi, sowie die vorderen und hinteren Wurzeln der zwei ersten Halsnerven. Die Nervenscheiden waren mit zahlreichen Leukocyten infiltriert, das Nervenparenchym selbst intakt. Die beiden Autoren erklärten jedoch diesen Fall als kompliziert durch das zufällige Hinzutreten einer bulbo-spinalen Meningitis.

I'on den tierexperimentellen Arbeiten, die zur Stütze der Theorie von dem primär zentralen Angriffspunkt des Diphtherietoxins dienen können, seien hier nur die Untersuchungen von Foulerton und Thoms on erwähnt, die auf Grund ihrer Resultate für die meisten Fälle 
die centrale Natur der postdiphtherischen Lähmungen fordern, jedoch für einen Teil der Störungen - insbesondere die Gaumenlähmung eine periphere Alteration annehmen, wobei dem in der nächsten Nähe des diphtherischen Belags gebildeten konzentrierten Toxin eine große Rolle zugemessen wird. Rhigetti spritzte das diphtherische Toxin unter die Dura von Hunden und fand zahlreiche kleine Blutungen der Hirnrinde, manchmal auch der weißen Substanz; solche Herdläsionen sollen den wichtigsten Befund in dem komplizierten pathologischen Bilde darstellen, welches das Toxin hervorruft. Es wurden vornehmlich schwere Gefäßveränderungen beschrieben, Infiltrationen mit Plasmazellen und namentlich polynucleären Leukocyten, regressive und progressive Verïnderungen der Gefäßwandelemente und Thrombenbildungen; solchen Störungen des Gefïßsystems wurde die Hauptbedeutung für die beobachteten Herdläsionen beigemessen.

Andere Autoren nehmen einen vermittelnden Standpunkt ein und halten die zentralen und peripheren Veränderungen für an sich durchaus koordinierte Frscheinungen, so vor allem Pre ysz, Henschen, Luisad a c, die bei ihren Untersuchungen schwere zentrale wie periphere Störungen im Nervensystem nachweisen konnten, für welehe keine direkte gegenseitige Abhängigkeit zu postulieren war.

Schließlich sind noch die Untersuchungen von Hochhaus erwähnenswert, der in seinen Fällen von postdiphtherischer Lähmung neben intaktem Zentralnervensystem und wenig ausgesprochener interstitieller Neuritis eine vorwiegend im Zwischengewebe lokalisierte Myositis nachwies und daraus den Schluß zog, daß ,die diphtherischen Jähmungen, die bisher nur in den Nerven und im Rückenmark anatomisch nachweisbar waren, auch in den Muskeln ihrenSitz haben können."

Es ist schon oben betont, daß wir in unserem Falle die gefundenen Läsionen im zentralen wie peripheren Nervensystem gerade in Berücksichtigung des anatomischen Bildes für unabhängig voneinander halten müssen; freilich läßt sich die Frage, welcher Teil des Nervensystems zeitlich zuerst befallen wurde, schwerlich aus den gewonnenen Befunden beantworten; gerade in dieser Hinsicht verdienen die Liquorbefunde Römlilds bei ,postdiphtherischer Pseudotabes“ Beachtung, der auf der Höhe der Erkrankung im Lumbalpunktat mäßige Lymphocytose, vermehrten Wiweißgehalt und eine stark positive Nonnesche Phase-IReaktion fand, während die Verstärkung der hemmenden Wirkung des Lecithins im hämolytischen System ausblieb. Römhild machte - unseres Erachtens mit Recht - darauf aufmerksam, daß dieser Beobachtung eine gewisse prinzipielle Bedeutung zukommt, indem sie uns einen klinischen Hinweis auf das Befallensein des Zentralnervensystems gibt.

Schlir Blich sei nochmals als das bemerkenswerteste Resultat unserer 
anatomischen Untersuchung hervorgehoben, daß sich in diesem Falle das Gefäßsystem und die Lymphräume am meisten und wohl auch primär affiziert erwiesen. Vielfach machen die im Zentralnervensystem gefundenen Blutungen (vgl. auch Textfig. 3 u. 4) den Eindruck, als ob sie zunächst in die außergewöhnlich erweiterten Lymphräume der Gefäße stattfänden und erst sekundär und abnorm langsam das Nervengewebe in Mitleidenschaft zögen. Auch in den peripheren Nerven zeigten sich ganz entsprechende Veränderungen am Gefäß- und Lymphsystem. Ähnliche Flüssigkeitsansammlungen, wie sie sich hier an manchen Stellen beobachten ließen, wurden zwar auch bei anderen Affektionen in den endo- und perineuralen Lymphspalten festgestellt, u. a. von I) ürok im $N$. vagus bei Beri-Beri und von Doinikow bei experimenteller Bleineuritis des Kaninchens. Wie aber schon aus der Besprechung der einschlägigen Literatur hervorgeht, sind gerade in den diphtherischen Folgezuständen die Gefäße und Lymphscheiden sehr häufig der vornehmliche Sitz anatomischer Veränderungen, eine Tatsache, die ja zahlreiche Autoren dazu veranlal3t hat, darin die pathogenetische Grundlage der nervösen Erscheinungen zu erblicken. Auch Wertheim Salomonson bildet bei Besprechung der Neuritis diphtherica zwei Blutungen (nach Präparaten und Mikrophotogrammen von Prof. de Vries) ab, und P. Me yer lenkt die Aufmerksamkeit auf die in seinen Fiillen postdiphtherischer Lähmung gofundene „Neuritis nodosa“, auf jene Veränderungen des Endoneuriums, welche stellenweise zu förmlichen ödematösen Anschwellungen, zur Knotenbildung geführt haben. „,Mit unseren heutigen Ansichten über die Struktur der Nervenscheide stimmt die Annahme wohl überein, daß pathologische Exsudate in demselben sich an gewissen Punkten ansammeln und dort die erwähnten Knoten bilden können. Wollte man die Ergebnisse der Key - Retzi usschen Untersuchungen in ihrem ganzen Umfang berücksichtigen, so müßte man eigentlich mit Pierret und Sanclair die Möglichkeit zulassen, daß sich gerade in den serösen Bahnen des peripherischen und des zentralen Nervensystems der pathologische Vorgang der diphtherischen Lähmung abspielt."

Auch unser Fall wird dieser Ansicht eine Stütze sein. 1)aß eine solche theoretische Folgerung anatomischer Resultate eine leichtere Beantwortung der immer wieder sich aufdrängenden Frage ermöglicht, weshalb das Diphtherietoxin jene klinisch so wohlbekannten Wege geht und mit einer so auffallenden Auswahl ganz bestimmte Teil des Nervensystems befällt, möge hier nur kurz angedeutet sein.

Wenn wir also zusammenfassen, so zeigte sich in den untersuchten Gewebsteilen ein subakuter Rntzündungsproze 3 , dessen Ausgangspunkte vermutlich die Umgebungen der Gefäße sind, und zwar vorwiegend 
die Gefäße bestimmter für den Angriff des Diphtherietoxins elektiv disponierter Nervengebiete. Die Befunde ähneln den bereits von Buhl, Schweitzer, Oertel, Klebs, Pierret, P. Meyer u. a. erhobenen und geben jedenfalls denen recht, welche wie Me yer, Precsz, Henschen u. a. als Grund epidiphtherischer Lähmungen einen Krankheitsprozeß statuieren, welcher sowohl das zentrale als auch das periphere Nervensystem ergreift, bald mehr das zentrale, bald mehr das periphere betonend. Daß in unserem Falle die bulbären Kerngegenden etwas schwerer getroffen wurden, macht auch die anatomische Untersuchung wahrscheinlich.

\section{Literaturverzeichnis.}

Acker, Über Lähmungen nach Diphtherie. Deutsches Archiv f. klin. Med. 13, H. 4 u. 5 .

Babonneix, Paralysies diphter. et Neurite asc. 'Thèse de Paris 1904 und Revue mens. des malad. de l'enfance 22. 1904.

Baginsky, Abschnitt „Diphtheritis" in Nothnagels Handbuch $2,208$.

Bailly, Des paral. conséc. à quelques maladies aigues. Gaz. des hôp. 1872.

Buhl, Einiges über Diphtheric. Zeitschr. f. Biol. 3. 1867.

('harcot et Vulpian, Compt. rend. de la Soc. de Biol. 1862; zit. nach S pieler. 1) a maschino et Roger, Des altérations de la moëlle etc. Rev. de méd. 1881.

Déjérine, Recherches sur les lésions du syst. nerveux dans les paral. dipht.

Arch. de phys. men. et pathol. 5. 1878.

-..- De la paralysie ascendente aiguë. Thèse de Paris 1879.

-.- et Barth, Arch. de Physiol. 1880.

Doinikow, Beiträge zur Histologie und Histopathologie der peripheren Nerven. Histol. u. histopathol. Arbeiten 1911, 4, 3.

I) ̈̈rck, Untersuchungen über pathologische Anatomie der Beriberi. Zieglers Beiträge z. pathol. Anat. \&, Suppl. 1908.

Foulerton and Thom son, The causation of paral. in dipht. Edinb. med. Journ. 1902 ; zit. nach spieler.

Geyer, Über feinere Veränderungen im Nervensystem eines Falles von postdiphth. Lähmung. Jahrb. f. Kinderheilk. 43. 1896.

(ireenhow, Case of dipht. paral. 'Transact. of the clinic. soc. of London 1870. Harris, Postdiphterial Paralysis of the Palate. Brit. med. Journ. 10, 1. 1903. Henschen, Akute dissem. Rückenmarksklerose mit Neuritis nach Diphtherie. Fortschritte d. Med. 1896, Juli.

Hochhaus, Utber diphtherische Lähmungen. Virchows Archiv 124, H. 2. 1891. Kerschensteiner, Neuritis. Lubarsch-Ostertags allgem. Pathol. u. pathol. Anat.

11, 2. 1907.

Landouzy, De paralysies dans les malad. aiguës. Thèse de Paris 1880.

Leyden, Über Poliomyelitis und Neuritis. Zeitschr. f. klin. Med. 1880, 1.

Lorain et Lépine, zit. bei spieler.

Luisada, Beitrag zur Kenntnis der diphtherischen Lähmungen. Vortrag. Bericht im Jahrb. f. Kinderheilk. 1902, 55.

P. Me yer, Anatomische Untersuchungen über diphtherische Lähmungen. Virchows Archiv 85, 181. 1881.

Nordmann, Klinische Erfahrungen während der letztjährigen Diphtherie-Epidemie. Berl. klin. Wochenschr. 1912, 1470. 
Örtel, Über Diphtherie. Deutsches Archiv f. klin. Med. 1871, 8.

Pierret, zit. bei spieler.

Preysz, Beitrag zur Anatomie der diphtherischen Lähmungen. Pester med.-chir. Presse 1894.

Remak, Neuritis und Polyneuritis. Nothnagels Handbuch 9, III, 480.

Righetti, Sulle alterazioni dei centri nervosi provocati dellia tossina difterica. Contributo sperimentale all'anatom. patol. della encef. acute tors. Riv. di patol. nerv. et ment. 14, 9.

Römhild, Zur Klinik postdiphtherischer Lähmungen. Liquorbefunde bei postdiphth. Pseudotabes. Vortrag. Ref. Neurol. Centralbl. 1908.

Sinclair, Contribution à l'étude de la pathogénie des paral. diphtér. Thèse de Lyon 1880.

Spieler, Zur Pathogenese der postdiphther. Lähmungen und des Herztodes bei Diphtherie. Obersteiners Arbeiten 15, 512. 1907.

Wertheim-Salomonson, Neuritis und Polyneuritis. Lewandowskys Handb. der Neurol. 2. 1911.

Willbrandt. Saenger, Neurologie des Auges, 1, 237-247. 1900.

Wladimirow, zit. nach Kerschensteiner.

Zappert, Abschnitt "Nervenkrankheiten" in Pfaundler-Schlossmamns Handb. f. Kinderheilk. \&, 741 .

\section{Erklärung der Tafel I.}

Fig. 1. Perincurales Gefäß des X. facialis mit gewucherter Intima, von zahlreichen Lymphocyten und Polyblasten umgeben. Bielschowsky-Präparat. Mikrophotogramm.

Fiz. 2. P'athologische Lymphansammlung in einem Nervenbündel des $\mathrm{N}$. vagus mit zerstreut liegenden Körnchenzellen und Leukocyten. Zerstörung des Nervenparenchyms. Modifizierte Mallory-Härbung. Längsschnitt. Mikrophotogranm.

Fig. 3. Endoneurales Gefäß mit polynucleären Leukocyten völlig angefüllt. Aus dem Brustmark austretender Nerv. Das umgebende Nervenparenchym von pathologischer Lymphe aufgelockert, stellenweise auch hier poly. nucleäre Leukocyten. Modifizierte M allor y - Färbung. Mikrophotogramm. 